\title{
Affouillements consécutifs aux chapelets de tourbillons
}

\section{Scour due to eddy streams}

\author{
PAR W.A. VENIS, \\ INGÉNIEUR DE LA SECTION D'ÉTUDES THÉOHIQUES ET EXPÉRIMENTALES, \\ SERVICE DU DELTA, RIYSSWATERSTAAT, TA HAYE
}

\begin{abstract}
Le rétrécissement de l'ultime passe d'une digue de fermeture donne lien à la formation de rouleaux et de chapelets de tourbillons. Les affouillements causés par ces séries de tourbillons an fond ainsi qu'aux parafouilles, sont en général plus graves que ceux qui résultent des courants dits «purement bi-dimensionnels ».

D'antre part, ces affouillements dépendent de l'intensité du chapelet de tourbillons. L'effet de cette intensité a été le sujet d'études comparatives sur modèles à fond affouillable.

Grâce d̀ la rugosité du fond, la reproduction des chapelets de tourbillons est, dans certains cas, plus semblable sur les modèles distordus que sur les modèles non distordus.

Bien qu'il soit très difficile de déterminer l'échelle du temps pour les affouillements sur modèle distordu, on peut se former une idée d'une telle échelle en comparant les résultats du modèle à ceax du prototype.
\end{abstract}

The hed and toe wall scour caused by the eddy streams forming at the ultimate closing stage of a dyke is usually more serlous than that associated with "purely two-dimensional" flows. The degree of scour depends on the intensity of the eddy stream, the effects of which have been investigated in comparative studies carried out on movable-bed models. Owing to the bed roughness, more representative eddy trails are obtained with a distorded scale model in certain cases.

Despite the considerable difficulty of determining the time scale in scour tests on a movablebed model, some indication of its magnitude can nevertheless be obtained by comparing the model and prototype results.
Dans la communication précédente, vous avez eu un aperçu général des différentes méthodes de fermeture d'un estuaire, vues dans le cadre de l'écoulement bi-dimensionnel. Pourtant, si l'on se trouve placé devant le problème de réaliser un certain barrage, on ne peut pas se contenter de cette figuration simplifiée. Il faut alors considérer l'écoulement dans son ensemble. Et il apparaîtra que, surtout aux limites de la dernière passe à barrer, se formeront des chapelets de tourbillons qui peuvent attaquer le lit beaucoup plus profondément que ne le ferait l'écoulement bi-dimensionnel. Cela s'applique aussi bien aux matériaux à gros grains avec lesquels les seuils sous-marins ont été construits, qu'aux matériaux à grains fins dont le lit est constitué. $\mathrm{Je}$ vais vous expliquer tout cela à l'aide de quelques exemples.

Nous considérons tout d'abord le résultat d'une série d'expériences faites sur un canal de laboratoire dans lequel on avait construit un seuil noyé (fig. 1). D'un côté, ce seuil était limité par une tête de digue arrondie construite dans le canal et sur un talus ayant une pente de 1:10. Cette tête de digue arrondie provoquait un chapelet de tourbillons qui attaquaient le fond mobile en aval du barrage.

Dans les expériences, on a fait varier la hauteur du seuil et, en même temps, la vitesse moyenne sur le barrage était toujours adaptée selon la courbe indiquée sur le graphique de droite de la figure 2. La courbe de droite sur le graphique de gauche donne l'affouillement dans le modèle réduit, après une heure de débit, en fonction de la hauteur du seuil déversant. En comparaison, on voit aussi la courbe qui se rapporte à l'écoulement bi-dimensionnel, également après une heure de fonctionnement, avec les 

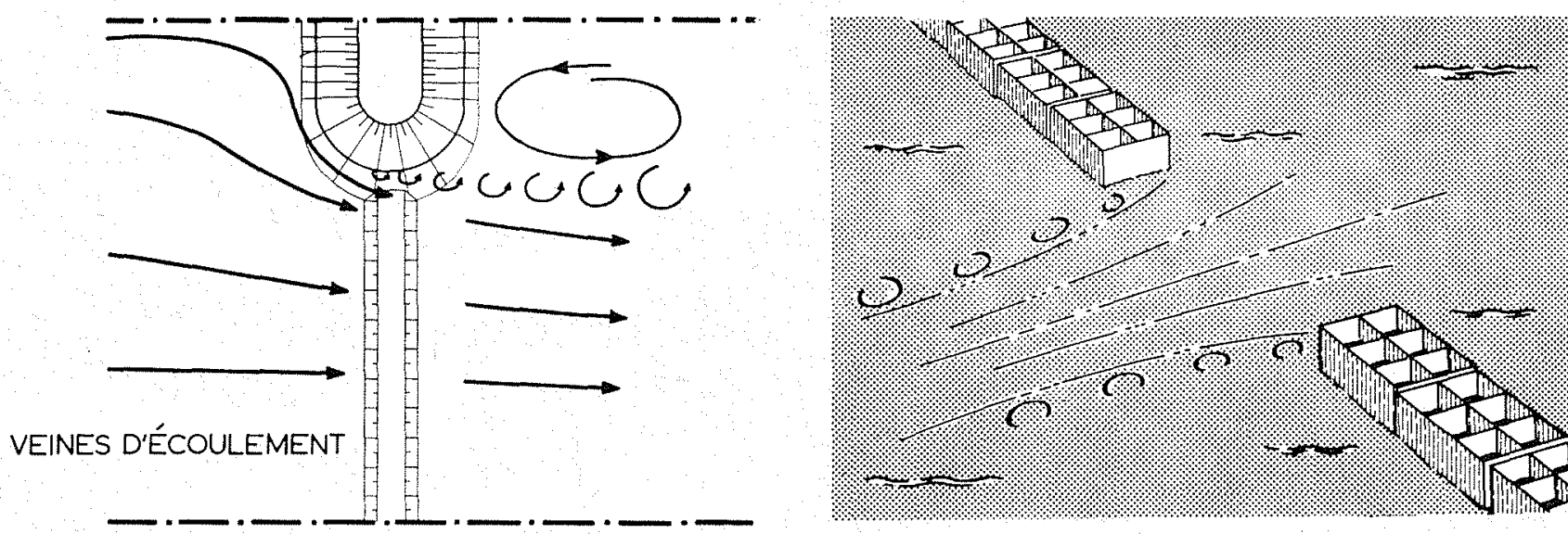

Frg. 3

Ecoulement, caissons pleins.

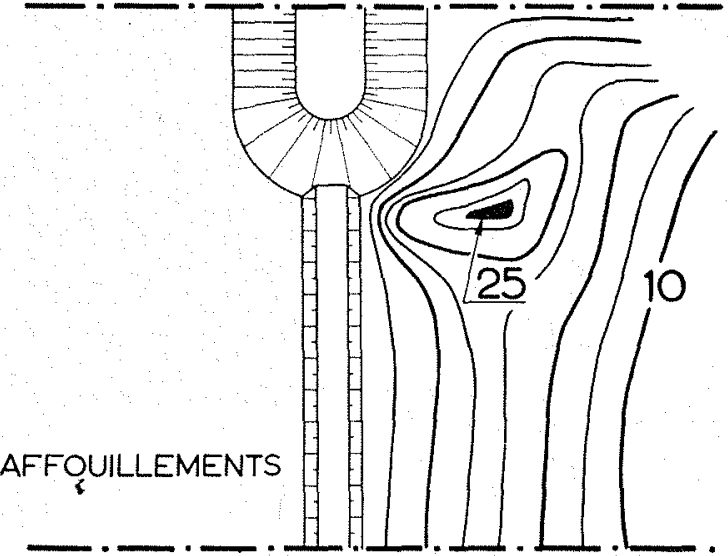

EIG. 1

AFFOUILLEMENTS APRÈS 1 HEURE D'ÉCOULEMENT

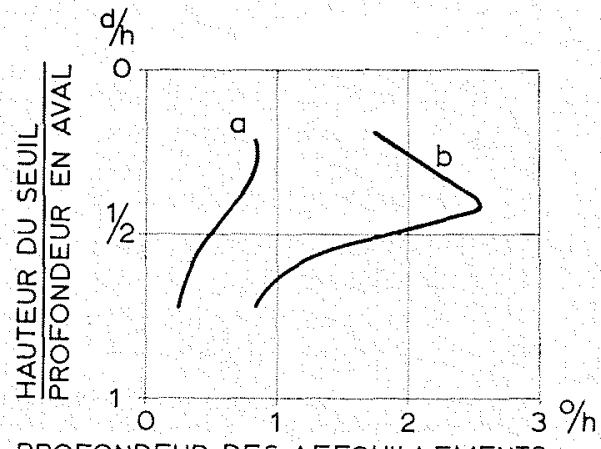

PROFONDEUR DES AFFOUILLEMENTS PROFONDEUR D'EAU EN AVAL

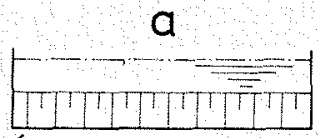

ÉCOULEMENT

BI-DIMENSIONEL

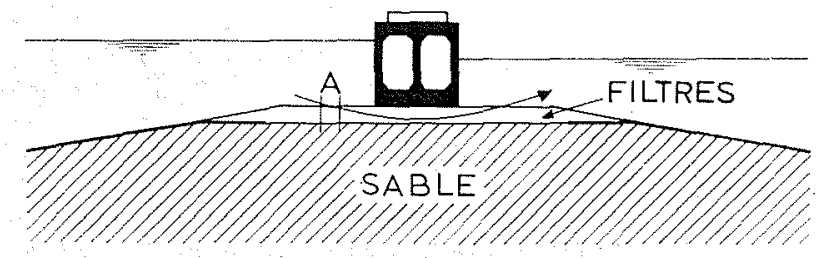

\%के ENROCHEMENTS GROS CALIBRE . DETAIL A ENROCHEMENTS PETIT CALIBRE GRAVIERS GROS GRAVIERS FINS

Fig, 4

Coupe du seuil du Veerse Gat.

VITESSE AU-DESSUS DU SEUIL DEVVERSANT

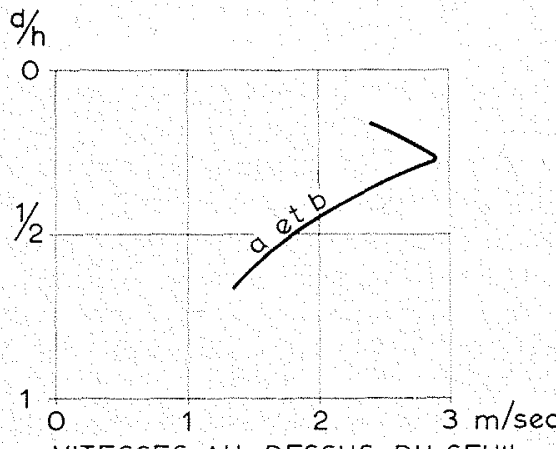

VITESSES AU-DESSUS DU SEUIL

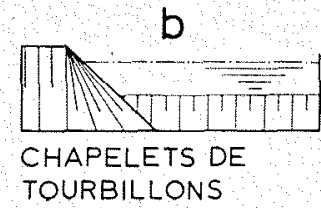

Fig. 2 
mêmes matériaux constituant le lit, c'est-à-dire de la bakélite finement moulue. 11 apparaît clairement ici que l'affouillement du lit est beaucoup plus grand quand il est dû̀ à un chapelet de tourbillons. Ces tourbillons ont été provoqués dans le modèle par une tête de digue arrondie ayant un talus à pente faible. Si nous avions eu affaire à une tête à parois verticales et se terminant à angles droits, la différence aurait été encore plus grande.

Dans la pratique, une tête de digue à parois verticales et à angles droits peut être formée par un caisson coulé dans la passe de fermeture (fig. 3). Les seuils sur lesquels ces caissons sont assis sont constitués par des enrochements. Dans les fermetures qui ont été réalisées jusqu'à présent, ces fondations sont formées par une couche de quelques mètres de grosses pierrailles sur un fond de sable.

Pour protéger le sable contre l'érosion, par suite du suintement à la base des caissons, les pierrailles ont été déversées comme un filtre de granulométrie graduée dont la couche la plus fine était en dessous et la couche la plus grosse au-dessus (fig. 4). On comprend que, par cette stratification, l'attaque de la couche supérieure pouvait facilement compromettre la stabilité de l'ensemble. La couche supérieure devait donc être en état de résister à l'attaque des chapelets de tombillons qui devaient se produire dès qu'un caisson serait coulé sur le seuil. Afin de vérifier quelles vitesses étaient encore admissibles dans la passe de fermeture dans de telles circonstances, avant que l'érosion de certains matériaux se produise, des expériences ont été faites dans un canal de laboratoire dans lequel un caisson avait été placé sur le seuil. Dans ces expériences on a utilisé trois matériaux différents pour constituer le seuil, à savoir : le basalte, l'enrochement et des cubes de béton (à l'échelle voulue).

Chaque fois que les matériaux du seuil commencaient à être entrainés par suite des tourbillons, la vitesse moyenne dans l'orifice disponible était mesurée. On voit sur le tableau (fig. 5) les résultats de ces mesures. Pour permettre une comparaison, j’ai également relevé ici les résultats des expériences sur modèle réduit faites en vue d'étudier la limite de stabilité de ces matériaux sur un seuil, dans le cas d'un écoulement bi-dimensionnel. On voit que les vitesses admissibles dans ce cas sont sensiblement diminuées de moitié, si hien que la présence d'un chapelet de tourbillons nous oblige à augmenter les dimensions des pierrailles du seuil bien plus qu'il ne l'aurait fallu sans la présence de ces tourbillons.

En parlant de l'affouillement, j'ai déjà dit qu'une paroi verticale à angles droits provoque une attaque plus violente qu'une tête de digue arrondie avec talus à pente faible. Il est également apparu que le chapelet de tourbillons provenant d'un caisson à pertuis ouverts est moins dangereux que celui qui provient d'un caisson plein. Cela signifie que l'intensité du chapelet de tourbillons joue un grand rôle. Il est pourtant très difficile de mesurer cette intensité, si bien que, pour le moment, on ne peut se baser que sur la capacité potentielle d'affouillement des tourbillons. C'est ainsi qu'à l'aide d'une étude comparée sur le modèle, on recherche de quelle manière l'affouillement peut ètre réduit en aval d'une tête de digue construite dans le courant. Dans le cas d'une fermeture par caissons, cette réduction est très difficile à réaliser étant donné qu'un caisson a presque toujours des parois verticales et que ce sont justement ces parois verticales qui provoquent les chapelets de tourbillons les plus intenses. Lorsque l'on projette une passe de fermeture qui sera comblée par remblayage graduel, on a beaucoup plus de liberté dans la configuration à donner aux parois des seuils sous-marins parce qu'il n'est pas indispensable que ceux-ci soient horizontaux sur une grande longueur.

Pour illustrer tout ceci, je puis vous montrer quelques résultats d'une série d'expériences qui ont été effectuées sur un modèle de la passe de fermeture septentrionale du Grevelingen. Comme M. Bischoff van Heemskerck vous l'a déjà dit, cette passe sera fermée l'année prochaine par remblayage graduel à l'aide d'un téléférique Blondin. L'été dernier, on a élevé un seuil de sable dans cette passe, jusqu'à une hauteur de six metres au-dessous du niveau moyen de la mer. Auparavant, la profondeur du chenal à cet en-

FiG. 5

Vitesse moyenne admissible dans le vertical. Profondeur d'eau $10 \mathrm{~m}$.

\begin{tabular}{|c|c|c|c|}
\hline & $\begin{array}{c}\text { BASALTE } \\
\varrho_{m}=2850 \mathrm{~kg} / \mathrm{m}^{3} \\
d_{m}=0,40 \mathrm{~m}\end{array}$ & $\begin{array}{l}\text { ENROCHEMENTS } \\
\mathrm{g}^{n}=2650 \mathrm{~kg} / \mathrm{m}^{3} \\
d_{m}=0,40 \mathrm{~m}\end{array}$ & $\begin{array}{l}\text { CUBES DE BÉTON } \\
\mathrm{g}^{m}=2200 \mathrm{~kg} / \mathrm{m}^{3} \\
\mathrm{~d}_{m}=0,40 \mathrm{~m}\end{array}$ \\
\hline Ecoulement bi-dimensionnel $\ldots \ldots \ldots \ldots \ldots \ldots$ & $7,50 \mathrm{~m} / \mathrm{s}$ & $7 \mathrm{~m} / \mathrm{s}$ & $5,5 \mathrm{~m} / \mathrm{s}$ \\
\hline Chapelets de tourbillons $\ldots \ldots \ldots \ldots \ldots \ldots \ldots \ldots$ & $3,75 \mathrm{~m} / \mathrm{s}$ & $3,50 \mathrm{~m} / \mathrm{s}$ & $2,75 \mathrm{~m} / \mathrm{s}$ \\
\hline
\end{tabular}



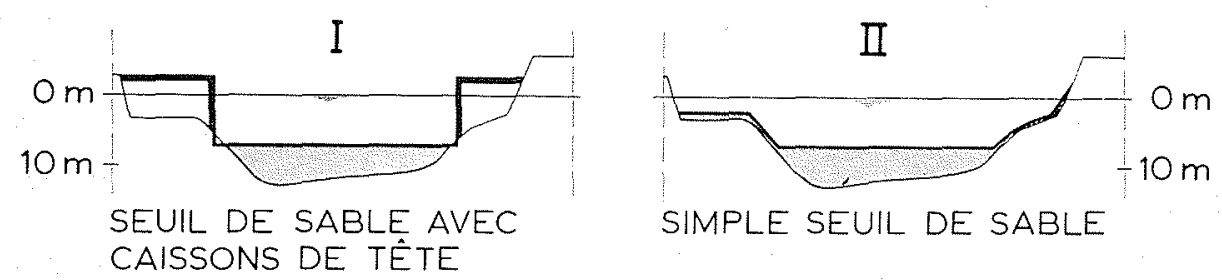

SIMPLE SEUIL DE SABLE

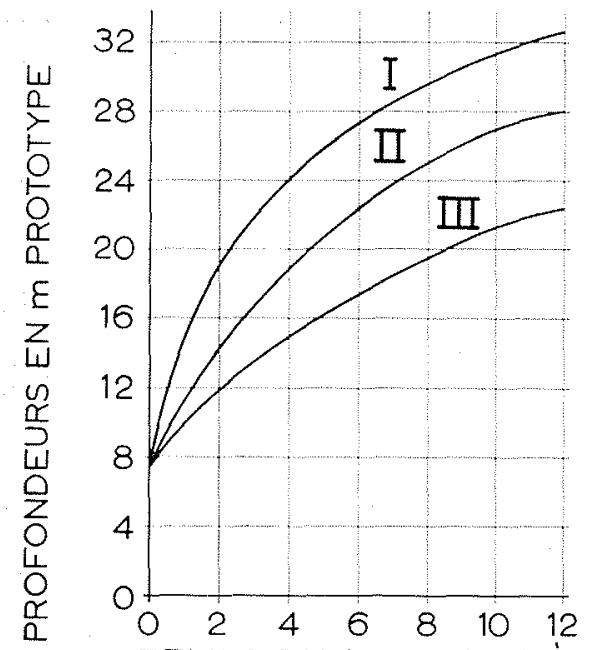

TEMPS EN h SUR MODÉLE RÉDUIT

\section{AFFOUILLEMENT MAXIMUM EN AVAL DE LA TÊTE DE DIGUE SEPTENTRIONALE}

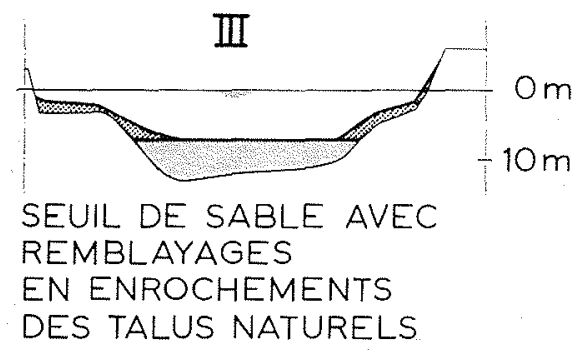

FIG. 6

Fermeture du Grevelingen, passe du Nord. Configuration de la passe de fermeture. Résultats des essais sur modèle réduit. droit était d'environ douze mètres. Ce seuil de sable est protégé au moyen de matelas de fascines. Etant donné que la fermeture n'aura lieu qu'au printemps de l'année prochaine, ce seuil sera exposé pendant huit mois environ aux marées et aux jusants. II ne faut pourtant pas que, pendant cette période, l'affouillement en aval de cette protection sous-marine soit trop grand.

Au cours des recherches, on s'est demandé si les affouillements pouvaient encore être limités en prenant des dispositions supplémentaires. II est apparu qu'un remblayage par enrochements, à l'endroit où le seuil de sable coupe le talus original du chenal, réduit considérablement l'affouillement. La figure 6 représente trois mém thodes d'exécution où, dans chaque cas, le seuil de sable a été élevé jusqu'à la même cote. Pour permettre une comparaison, j'ai porté plus bas l'accroissement en fonction du temps, de la plus grande profondeur de l'affouillement dû aux tourbillons qui se produisent en aval de la tête du môle septentrional.

C'est en effet à cet endroit que l'affouillement était le plus important dans le modèle. A còté de l'influence relativement grande qui est exercée par le remblayage partiel par enrochements des talus naturels du chenal, on voit maintenant clairement dans quelle mesure l'affouillement augmente si nous donnons à la culée des parois verticales, à angles droits. La configuration plus libre qui est rendue possible par une fermeture graduelle, peut donc être d'une grande importance pour réfréner les tourbillons. Dans le cas d'une fermeture à l'aide de caissons, on peut essayer de limiter les affouillements en retardant le plus possible la mise en place des parois verticales.

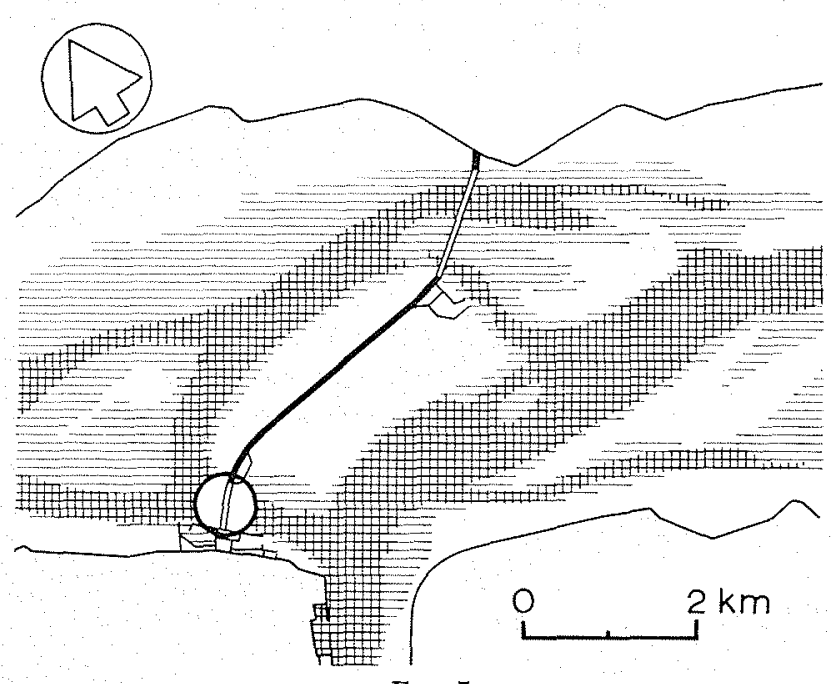

FIG. 7

Passe de fermeture méridionale du Grevelingen. 

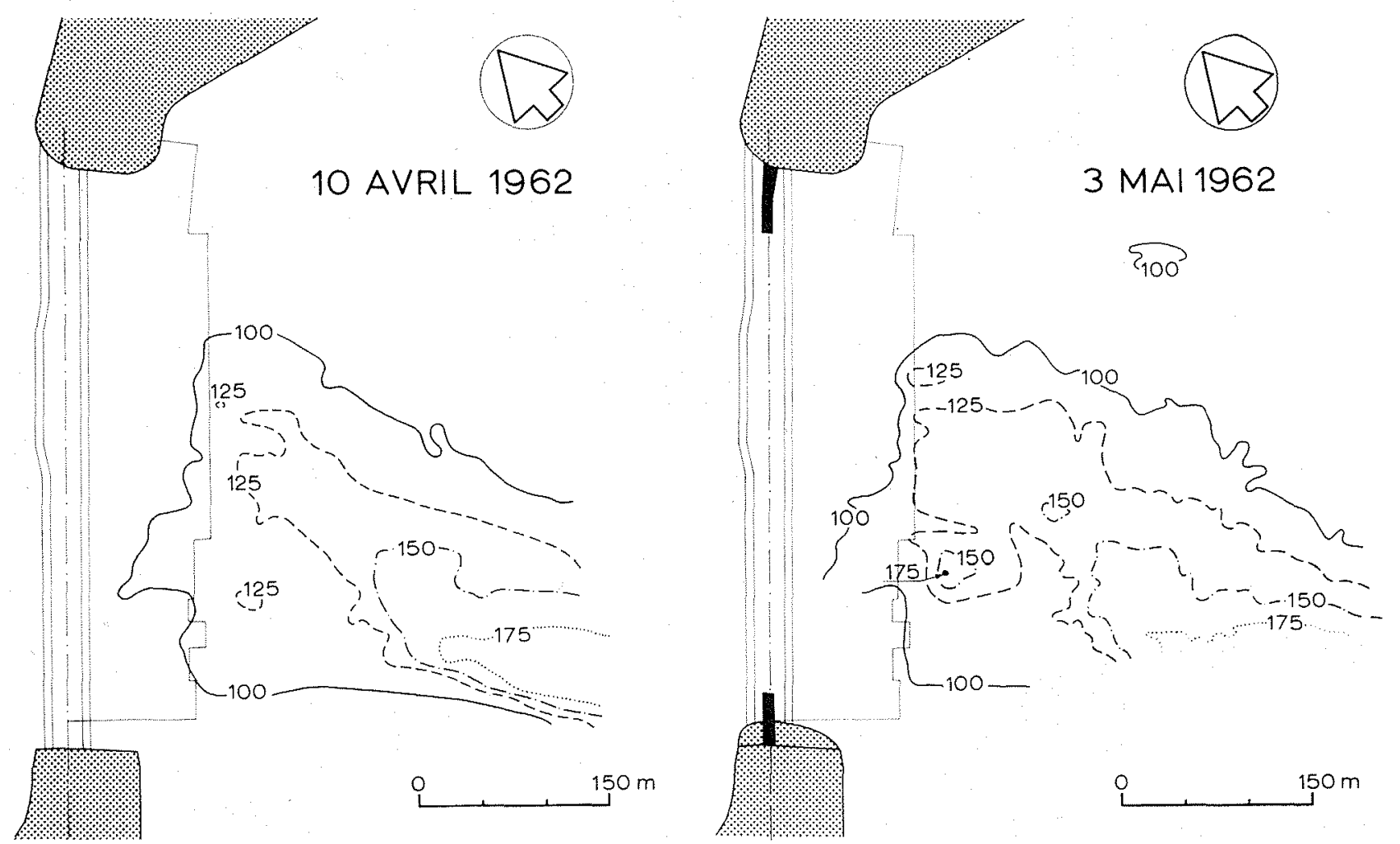

FIG. 8

Affouillements dus au caisson de tête méridional à la passe de fermeture du Grevelingen.

Cette méthode a été appliquée lors de la fermeture de la passe dans le chenal méridional du Grevelingen, fermeture qui a eu lieu au début de cette année (fig. 7). Dans ce chenal également, on avait d'abord élevé un seuil de sable qui atteignait une hauteur d'environ sept mètres audessous du niveau moyen de la mer. Dans ce cas, la fondation sur laquelle les caissons étaient assis était constituée par une masse de pierrailles, d'environ deux mètres d'épaisseur et composée de couches de gravier et d'enrochements. La fermeture elle-même eut lieu à l'aide de ce que nous appelons des « caissons standard». Ce sont des blocs de béton rectangulaires creux, dont les parois pleines ont onze mètres de longueur et six mètres de hauteur. Ces petits caissons sont très maniables, mais ils exigent néanmoins une culée verticale pour permettre le raccordement à la digue existante.

Les culées qui, ici également, étaient formées par des caissons standard, ont été placées, le 10 avril, alors que la phase de fermeture proprement dite commencait le 3 mai. Dans cette courte période de trois semaines, pendant laquelle les culées sont restées dans le chenal, il s'est encore produit, à l'est de la tête de digue méridionale, un approfondissement d'environ sept mètres. On voit une fois de plus un exem- ple de l'influence d'une paroi verticale continue. Qu'il n'y ait pas eu d'affouillement en aval de l'autre tête de digue et à l'ouest, cela peut s'expliquer par la répartition des vitesses du courant (fig. 9).

D'une part, la vitesse du courant dans le sens ouest-est était ici plus grande que dans le sens est-ouest; elle était en moyenne de $1,70 \mathrm{~m} / \mathrm{s}$ contre $1,40 \mathrm{~m} / \mathrm{s}$. D'autre part, le courant ouest-est était plus concentré le long de la rive méridionale, alors que le courant est-ouest était réparti sur toute la largeur de la passe de fermeture (fig. 9).

Dans la fermeture du Veerse Gat, qui a eu lieu l'année dernière, les circonstances étaient toutes différentes. Cette passe a été fermée à l'aide de ce que l'on appelle des caissons à pertuis. Ceux-ci ont des dimensions beaucoup plus grandes que les caissons standard. Ils ont environ $45 \mathrm{~m}$ de longueur et $20 \mathrm{~m}$ de hauteur; en outre, après coulage dans la passe de fermeture, ils peuvent être presque entièrement ouverts de façon à permettre le libre passage des marées. Cependant ils nécessitent également un caisson-culée vertical pour permettre leur raccordement à la digue existante. Cette tête de digue a été formée ici par des caissons Phoenix AX. On connait bien ce type de caissons qui, 


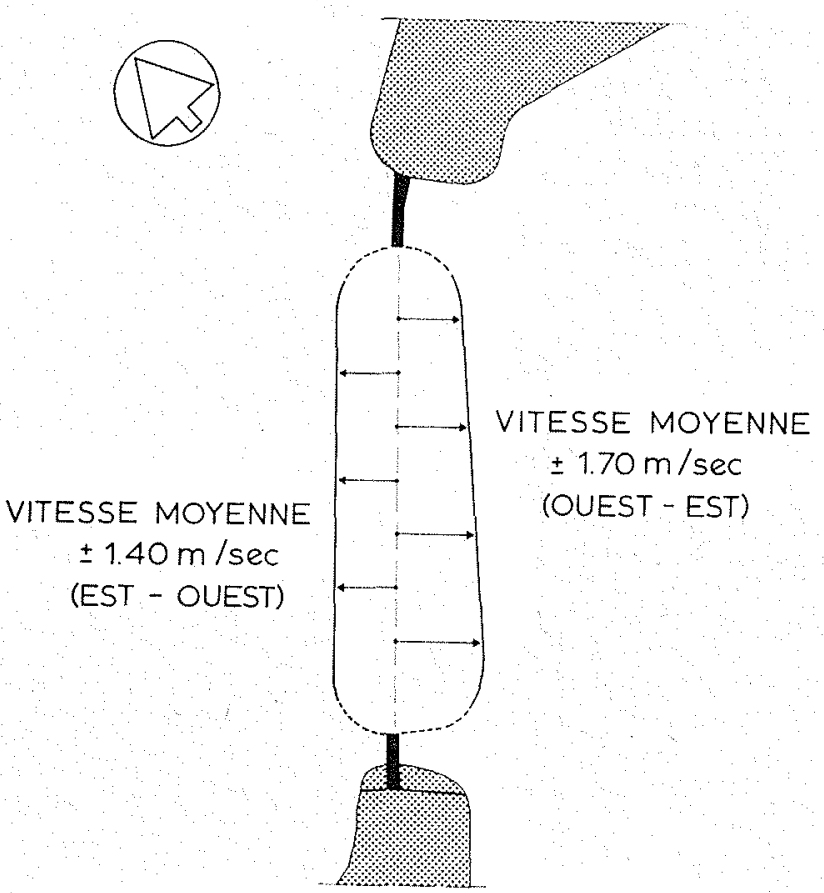

Fig. 9 Passe de fermeture méridionale du Grevelingen. Répartition des vitesses.
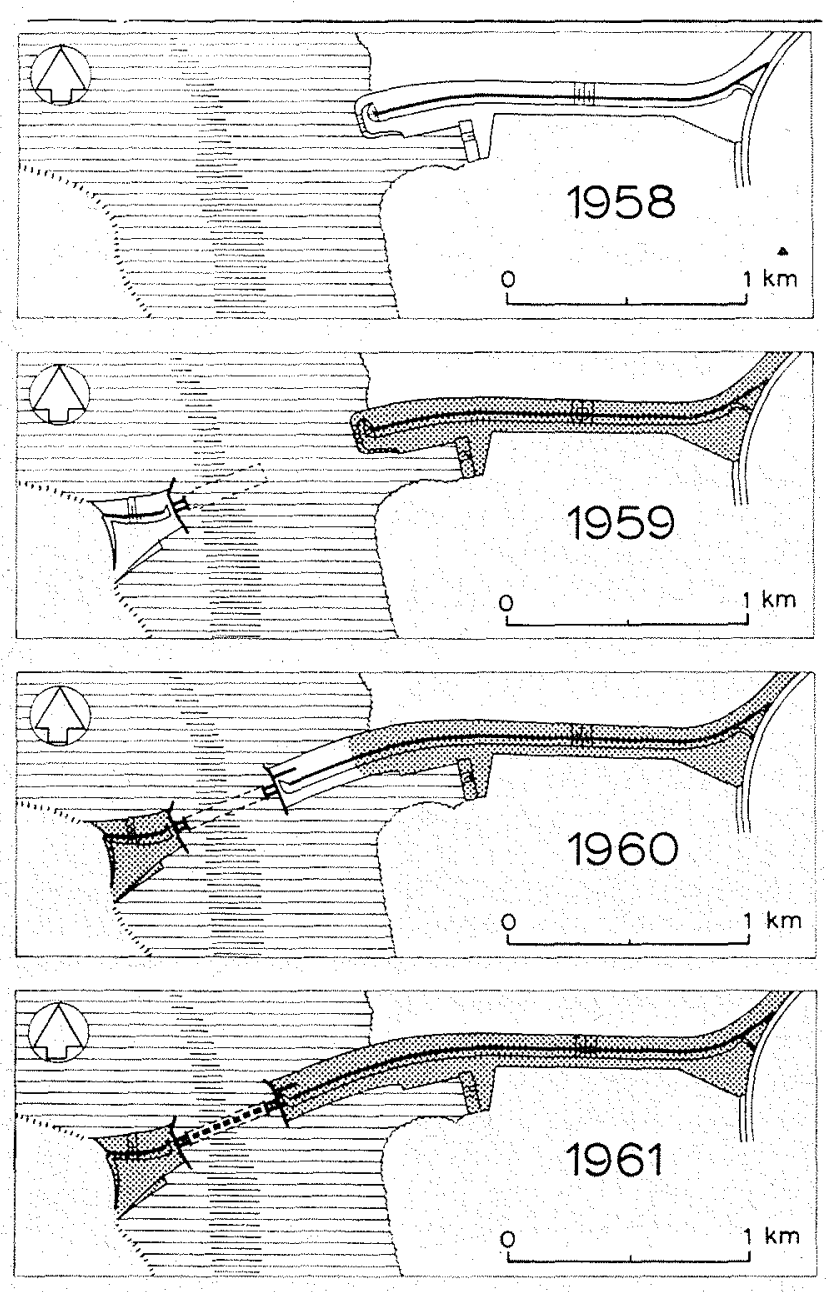

lors du débarquement, a servi à la construction de la tête de pont d'Arromanches.

Les travaux dans le Veerse Gat ont commencé en 1958 par la construction d'un troncon de digue sur le «Onrustplaat». En 1959, la tête de digue occidentale fut construite et en 1960, la tête de digue orientale. Par la construction de ces têtes de digues avec troncons de raccordement, une partie considérable du profil d'écoulement fut obturée (fig. 10).

Je puis illustrer cela à l'aide de la répartition des vilesses dans le Veerse Gat avant le commencement des travaux (fig. 11). Le courant de jusant est concentré dans le chenal profond et passe approximativement par l'orifice de la passe. Au contraire, le courant de flot est réparti beaucoup plus régulièrement sur toute la largeur du Veerse Gat. C'est surtout par suite de la construction de la tête de digue orientale qu'une grande partie du profil disponible pour le flot fut coupée.

Sans précautions supplémentaires, après l'achèvement de la construction des deux têtes de digue, l'écoulement au moment du flot, serait celui qui est schématisé sux la figure 12 à gauche. On voit que la plus grande contraction a lieu bien en aval de la passe de fermeture, tandis que le courant principal est flanqué de chapelets de tourbillons très violents provenant des caissons-culées. La figure de droite présente une schématisation de la forme des têtes de digue telles qu'elles sont construites en réalité. L'écoulement est devenu beaucoup plus favorable, la contraction maximale se trouve maintenant à peu près dans la passe elle-même, alors que les chapelets de tourbillons sont également beaucoup moins violents et ont effectué un plus grand parcours avant de s'attaquer au lit, en aval du matelas de fascines.

Les petites contre-digues, de chaque côté de la passe de fermeture, les « oreilles》 comme nous les appelons, sont difficiles et coûteuses à réaliser. Contrairement à ce qui s'est passé au Grevelingen, on a été obligé d'effectuer toutes les constructions des têtes de digues dans les deux années qui ont précédé la fermeture. Vous comprenez que l'on préfère effectuer la fermeture le plus tôt possible dans la saison ouvrable et c'est la raison même pour laquelle on tâche de se débarrasser de tous les travaux supplémentaires. La conséquence en fut que la passe de fermeture était déjà terminée neuf mois avant la fermeture réelle.

La situation au moment du flot est tracée ici. Comme je l'ai déjà expliqué, c'est le cas

Fra. 10 Veerse Gat.

Ordre de construction de la digue de fermeture. 


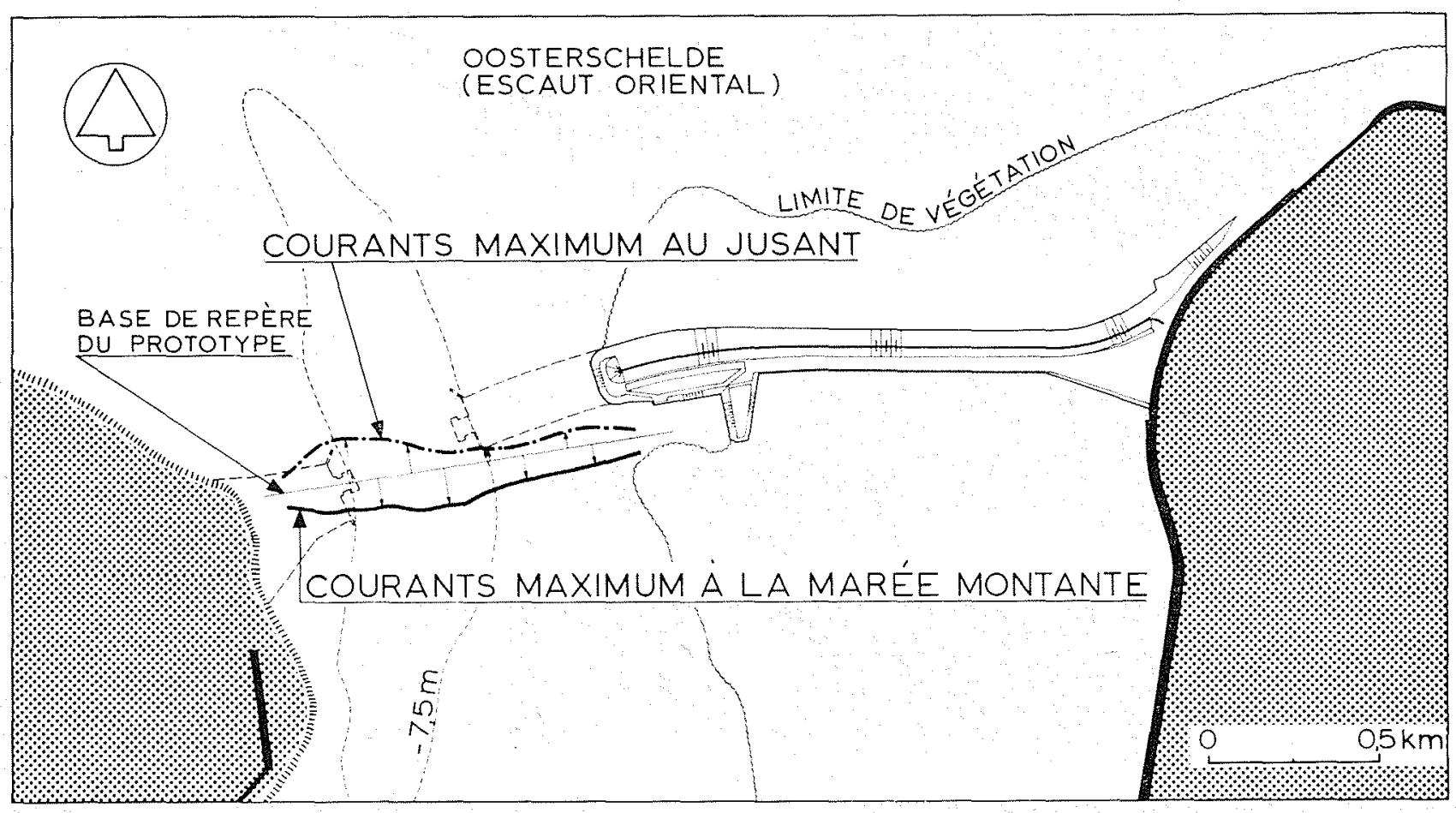

FIG. 11

Veerse Gat. Répartition des vitesses.

oì la perturbation était la plus forte et où les vitesses maximales étaient les plus grandes; atteignant une moyenne de $2 \mathrm{~m} / \mathrm{s}$, alors que la vitesse maximale du jusant dans des circonstances normales était d'environ $1,60 \mathrm{~m} / \mathrm{s}$.

De chaque côté du seuil déversant qui devait servir d'assise pour les caissons de la passe de fermeture, des fosses ont été draguées au cours des années précédant la fermeture (fig. 13). Ces fosses atteignaient une profondeur d'environ vingt mètres au-dessous du niveau moyen de la mer. Le fond original du chenal se trouvait ici à environ onze mètres de profondeur. Le but de ce dragage était de permettre au courant de se disperser en aval de la passe de fermeture, de façon à réduire l'attaque du lit au-delà du matelas de fascines. Si l'on applique un tel procédé, il faut soupeser d'une part l'avantage d'un affouillement provoqué sous contròle et d'autre part l'inconvénient de courir le risque, dans le cas où il se produirait quand même un affouillement supplémentaire, de tomber dans des couches de sable mal tassé, situées à une plus
FIG. 12

Veerse Gat Aménagement de la passe de fermeture.
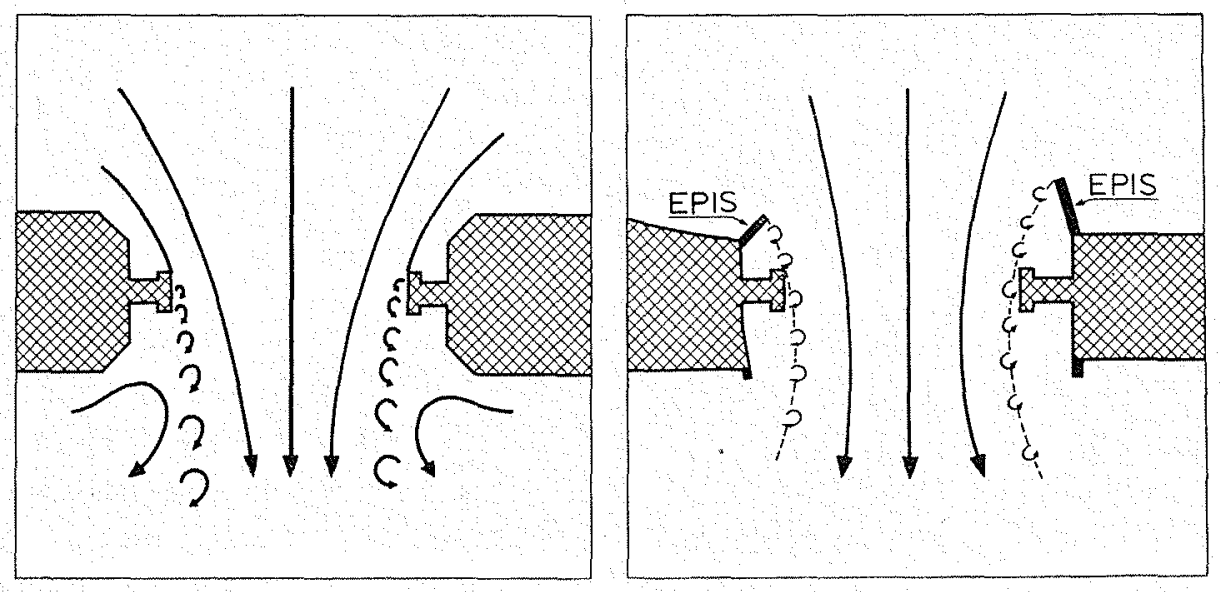


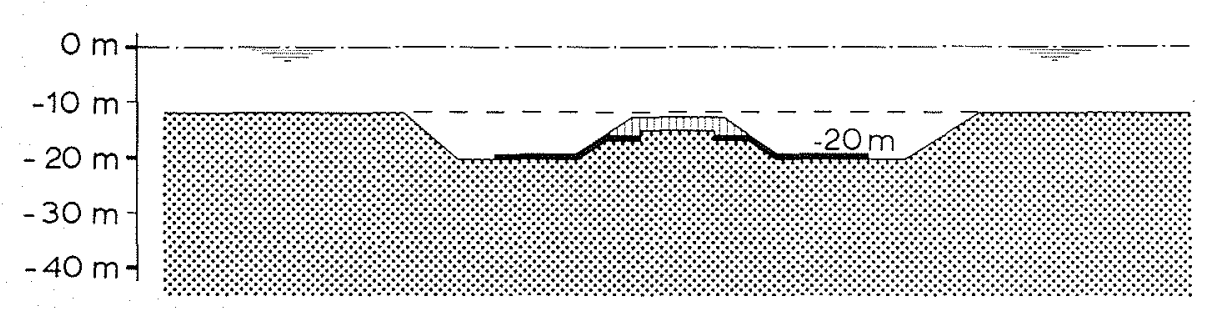

FIG. 13

Veerse Gat.

Dragages des deux côtés du seuil,

$400 \quad 300 \quad 200 \quad 100 \quad 0 \quad 100 \quad 200 \quad 300 \quad 400 \mathrm{~m}$

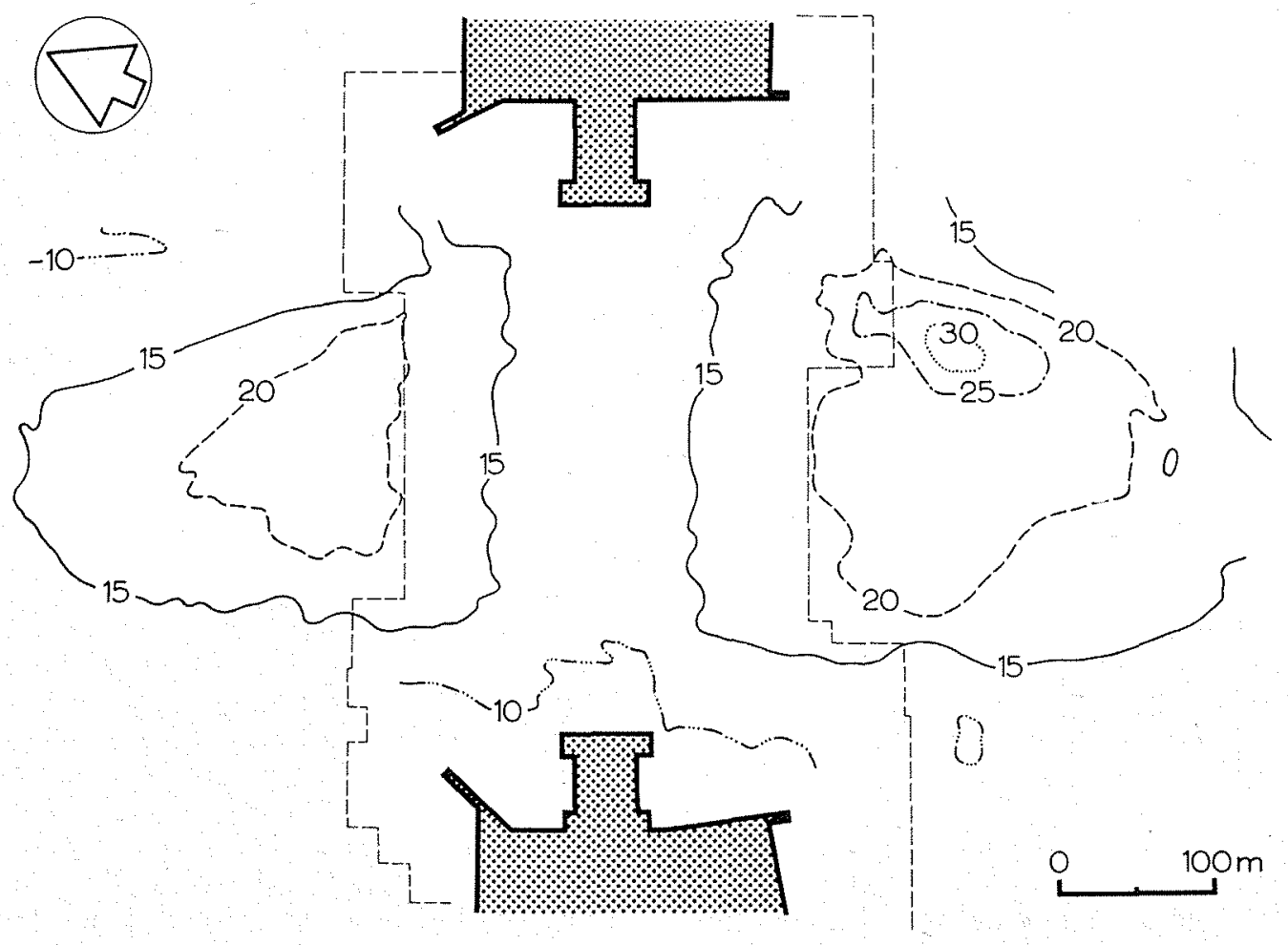

FIG. 14

Veerse Gat. Topographie des courbes de profondeur janvier 1961.

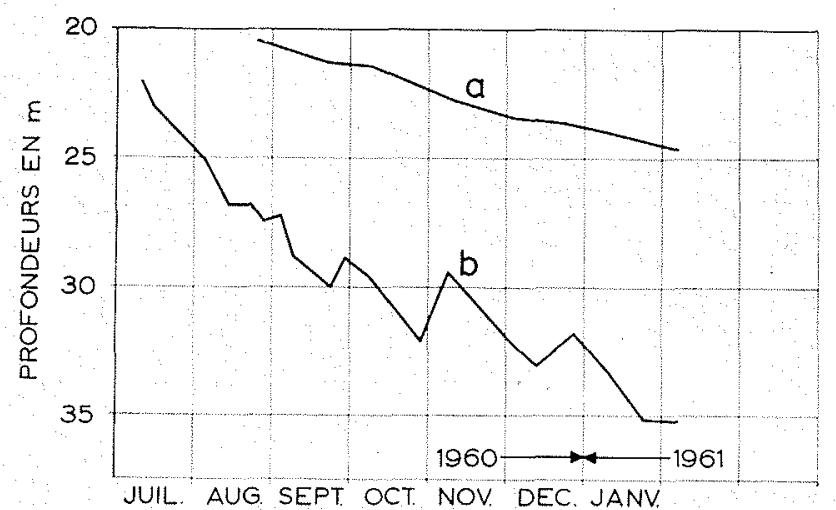

Fig. 15

Veerse Gat.

Affouillement maximal en fonction du temps.

a) Au milieu et en aval du seuil;

b) Comme suite du chapelet de tourbillons de la tête de digue orientale. 
grande profondeur. Dans le Veerse Gat on n'avait heureusement pas à craindre ces couches de sable mal tassé. Aussi bien par ce dragage que par la construction des « oreilles » qui devaient réduire considérablement l'intensité des chapelets de tourbillons, nous pouvions espérer que l'affouillement resterait dans des limites très raisonnables. De la carte des sondages qui a été dressée environ six mois après que la passe de fermeture était prête, il ressort que les mesures prises ont en effet restreint l'affouillement, à une exception près (fig. 14). A notre grande surprise, le chapelet de tourbillons qui s'était produit près de la tête de digue orientale au moment du flot a pourtant miné sérieusement le sous-sol.

Sur la figure 15 on voit représentée l'évolution de la profondeur de cet affouillement en fonction du temps. Pour faire une comparaison, on a également tracé, en fonction du temps, la courbe des profondeurs de l'affouillement qui s'est produit en aval des matelas de fascines, au milieu de la passe de fermeture, là où l'écoulement était donc approximativement bi-dimensionnel. On voit donc que même un chapelet de tourbillons dont l'intensité est réduite peut encore être très dangereux si les circonstances dans lesquelles il se produit durent assez longtemps.

A l'aide de quelques exemples, j'ai essayé d'expliquer ici que, si une étude bi-dimensionnelle peut rendre de grands services pour un projet en général, il n'en reste pas moins vrai que, dans l'étude détaillée de chaque passe de fermeture, il faut accorder une grande attention aux effets locaux tri-dimensionnels qui peuvent exercer une influence très importante sur l'ensemble. Cela s'applique aussi bien à la forme à donner à nos constructions qu'à la cadence à laquelle elles doivent être réalisées. Les études hydrauliques sur modèle réduit nous sont heureusement d'une grande utilité. Cependant, si l'on veut se fier aux résultats de ces études, il faut avoir la certitude que le modèle correspond le plus fidèlement possible au prototype. Cela exige tout d'abord que l'écoulement soit reproduit très exactement dans le modèle. La plus grande difficulté à laquelle on se heurte pour satisfaire à cette première exigence est de pouvoir reproduire à l'échelle exacte la rugosité du fond. Dans les études des aflouillements sur modèle réduit, nous rencontrons encore une autre difficulté. Voulons-nous savoir par exemple si certaines constructions peuvent se maintenir quelques semaines ou si elles peuvent résister sans inconvénient pendant plusieurs années, il est alors indispensable de pouvoir interpréter les expériences sur les affouillements également en fonction du temps. Cette interprétation n'est possible que si nous connaissons l'échelle du temps de l'usure du fond dans le inodèle. Ce problème est actuellement à l'étude et, dans une des communications suivantes, mon collègue du laboratoire de Delft y reviendra de façon plus détaillée. Mais il ne parlera que des résultals connus, jusqu'à présent, de la recherche systématique sur des écoulements bi-dimensionnels. On ne sait pas encore dans quelle mesure ces résultats sont applicables aux affouillements dus aux chapelets de tourbillons. Pour se faire une idée de l'échelle du temps, le seul

FIG. 16

Veerse Gat. Extension des modèles réduits.

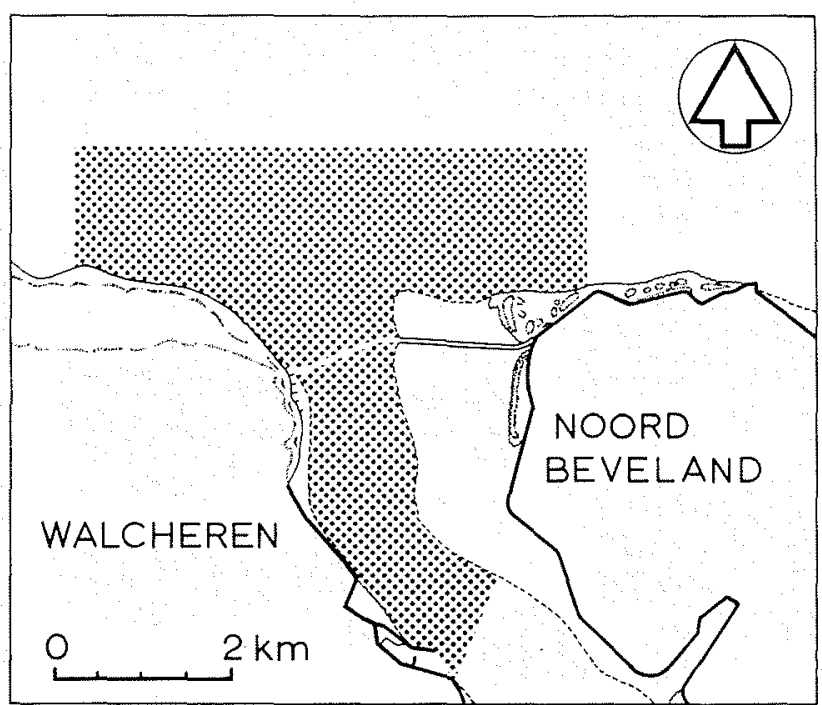

Modèle à distorsions.

Echelle horizontale $1 / 150$; verticale $1 / 50$.

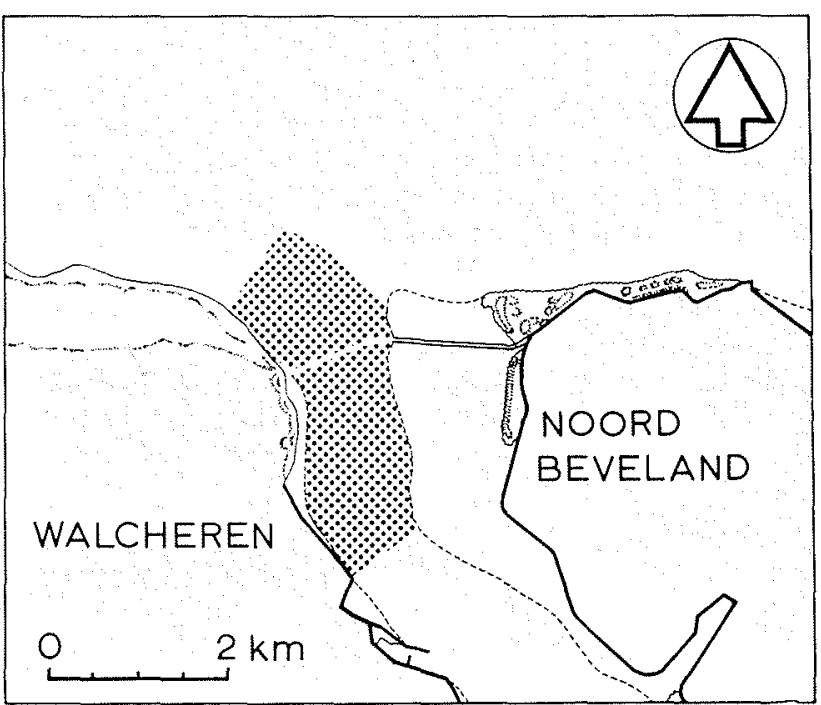

Modèle non distordu. Echelle 1/50. 
moyen dont nous disposons pour le moment est la comparaison directe du modèle avec le prototype et cela n'est évidemment possible que si des affouillements se sont effectivement produits dans le prototype. Pour une bonne recherche des affouillements sur un modèle réduit, il faut donc satisfaire au moins à deux conditions : premièrement l'écoulement dans le modèle doit concorder avec ce qu'il est dans le prototype et deuxièmement il faut connaître l'échelle du temps.

Le mouvement de l'eau, aussi bien vertical qu'horizontal, joue un très grand rôle, surtout lors de l'affouillement en aval d'un seuil sousmarin. Pour l'étude des affouillements, on donnera donc en principe la préférence à un modèle non distordu. Dans un modèle à distorsion, le mouvement horizontal de l'eau est reproduit à une autre échelle que le mouvement vertical et cela a pour conséquence que l'écoulement vertical ne peut pas y être très bien représenté.

L'expérience nous a pourtant appris que l'écoulement horizontal est souvent beaucoup mieux représenté dans un modèle à distorsion que dans un modèle non distordu. On peut donc se servir d'un modèle à distorsion quand le mouvement vertical de l'eau est d'importance secondaire. Cela va même si loin que les affouillements provoqués par les chapelets de tourbillons ayant un axe vertical peuvent très bien être reproduits dans un modèle à distorsion. Le chapelet de tourbillons fait partie de l'écoulement en plan horizontal et les affouillements qui en résultent dépassent de beaucoup ceux d'un écoulement purement bidimensionnel.

Je vais illustrer ceci à l'aide d'un exemple (fig. 16). Pour cela, je reviens au Veerse Gat. Autrefois, on en a construit deux modèles : un modele à distorsion à l'échelle de 1:150 en plan horizontal et de 1:50 en plan vertical et un modèle non distordu à l'échelle de 1:50. Comme vous le voyez, le modèle à distorsion représentait une plus grande surface que le modèle normal. Ces deux modèles avaient été construits dans le laboratoire en plein air de « de Voorst». Le sol, qui $y$ est très dur et mal compressible, se compose d'une sorte de sable fin mélangé à du limon. En construisant les modèles, on a pensé que la rugosité de ces matériaux convenait très bien pour une reproduction du prototype à l'échelle. de 1:50. Lors de l'étalonnement du modèle normal, on s'est pourtant rendu compte que la rugosité du modèle était légèrement trop grande. Le modèle à distorsion a paru comme étant trop lisse et il fut amené à la bonne échelle de résis-

FIG, 17 en prototype et en modèle réduit.

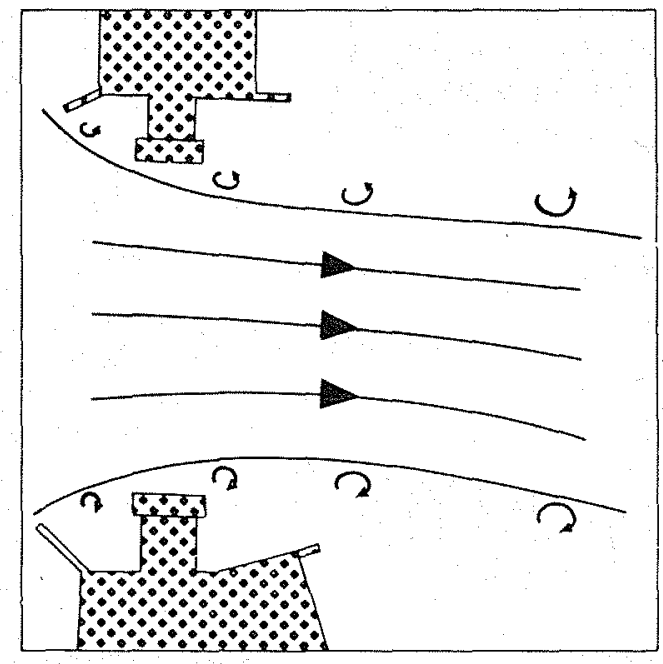

Prototype.

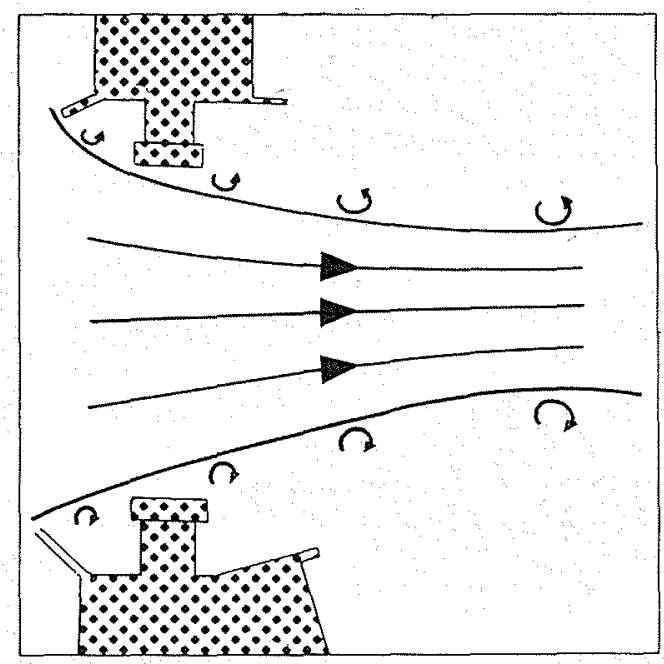

Modèle à distorsion.

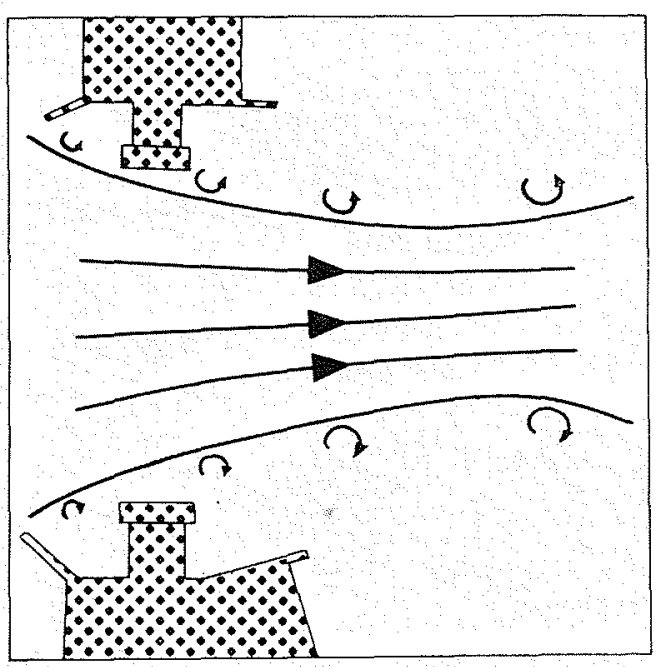

Modèle non distordu. 
Fic. 18

Veerse Gat Comparaison des affouillements en prototype et en modèle réduit.
PROTOTYPE

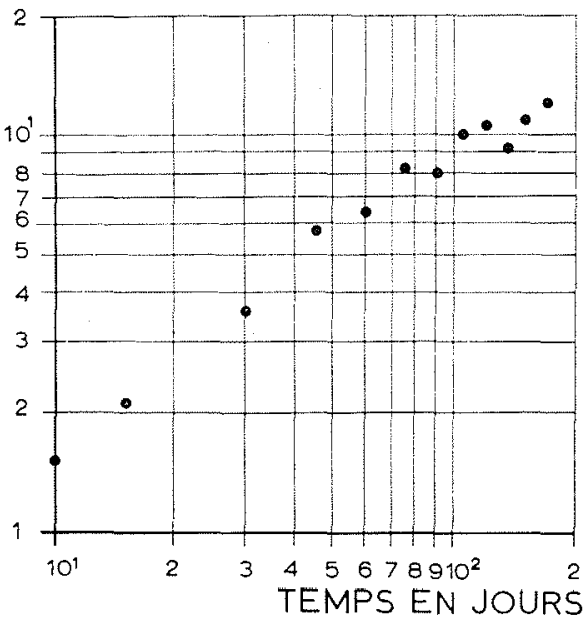

MODĖLE RÉDUIT

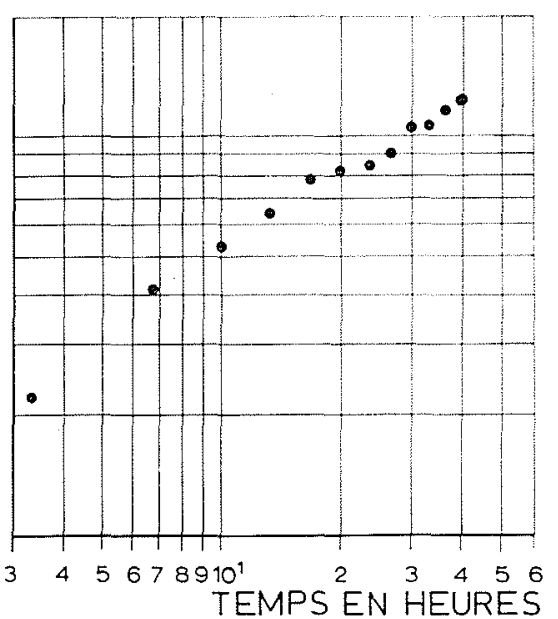

tance par l'introduction d'une rugosité artificielle. Les différences du modèle normal avec le prototype ressortirent très clairement au cours de la réalisation de l'obturation de la passe de fermeture.

Aux moments des vitesses maximales des courants, on a pris des photographies aériennes de l'écoulement dans le prototype. Puis on les a comparés avec les photographies analogues des deux modèles. Il en est apparu que le modèle à distorsion représentait très bien l'écoulement alors que, dans le modèle normal, le courant passait trop en biais par l'orifice. Malheureusement, ces photos ne se prêtent pas à la projection et j'ai fait un croquis de la schématisation de l'écoulement (fig. 17).

J'ai déjà montré que, dans la passe de fermeture, il s'est produit un affouillement considérable au sud de la culée orientale de NordBeveland (Cf. fig. 14). Comme la configuration de l'éconlement se reproduisait si fidèlement dans le modèle à distorsion, on se décida à l'utiliser pour les recherches relatives aux affouillements. Comme matériaux affouillables, on a choisi de la bakélite finement moulue. Pour parvenir à entraîner ces matériaux, on sera le plus souvent obligé d'augmenter les vitesses dans le modèle, en rapport avec l'échelle des vitesses d'après le nombre de Froude. Dans ce modèle, l'échelle des vitesses de Froude était de $\sqrt{50}$, c'est-à-dire : 7,1. Les expériences d'affouillement ont été faites d'après une échelle des vitesses de 6,6 , donc comme on le voit, avec une distorsion négligeable de l'écoulement.

Le résultat des expériences est représenté sur la figure 18, où, à échelle logarithmique, l'évolution du point le plus profond de l'affouillement a été portée en fonction du temps, aussi bien pour le modèle que pour le prototype. La concordance est très bonne et, sur cette base, on a admis que pour une échelle de la vitesse de 6,6 , une heure d'écoulement dans le modèle équivalait à 4,5 jours dans le prototype. Une reproduction du point le plus profond ne suffit évidemment pas; la forme de l'affouillement ainsi que la quantité des matériaux entraînés doivent encore concorder.

Une comparaison des cartes de sondage dans le prototype et le modèle sur des périodes concordantes montre que la forme de l'affouillement est relativement bien reproduite, mais que, dans le modèle, le point le plus profond s'est légèrement déplacé vers le centre de la passe de fermeture (fig. 19). Il faut probablement attribuer cela à une conséquence de la rugosité trop faible de la bakélite; il s'ensuit que, dans le modèle, il s'est produit une contraction légèrement trop grande en aval de la passe de fermeture. La similitude n'est donc pas parfaite, mais elle était pourtant suffisante pour pouvoir interpréter les expériences que nous avons faites ensuite en plaçant les caissons à pertuis. En se basant sur les différences qui ont été trouvées entre l'affouillement provoqué par les caissons pleins et celui provoqué par les caissons à pertuis, on décida par exemple d'ouvrir complètement les caissons immédiatement après leur coulage. Le programme de fermeture établi sur la base de ces expériences a été un tel succès, que lors du coulage des caissons, il ne s'est plus produit d'affouillement supplémentaire. En ce qui concerne le problème de l'échelle du temps, il ne s'est pas révélé de nouveaux points de vue.

La difficulté dans un modèle à distorsion est donc que, si l'on veut définir une échelle du temps, il faut que des affouillements se soient d'abord produits dans le prototype. Il serait évidemment avantageux si les résultats des échel- 

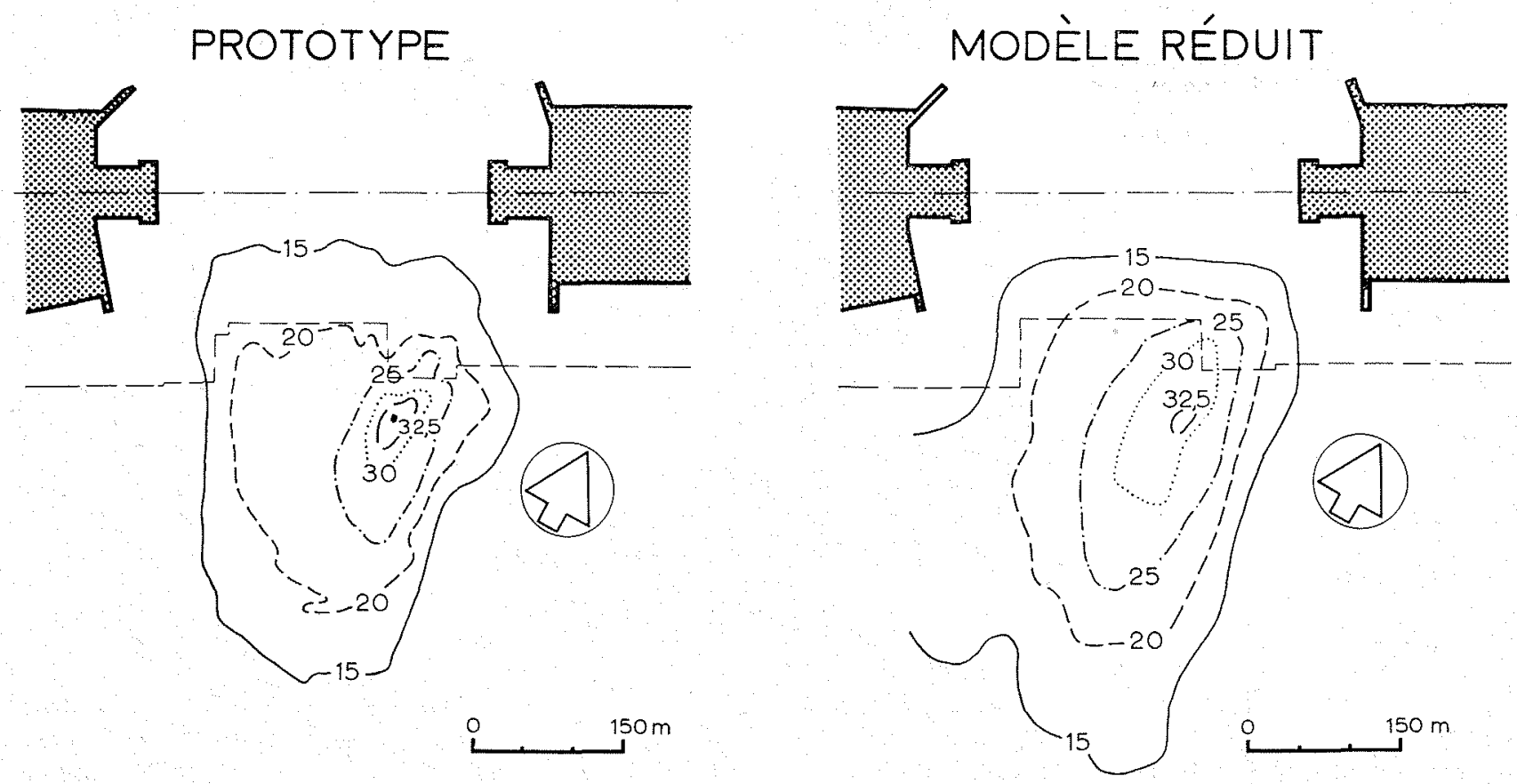

Fig. 19

Veerse Gat. Comparaison des courbes de profondeur en prototype et en modèle réduit.

les du temps dans le cadre de l'écoulement bidimensionnel, résultats dont on entendra parler tout à l'heure, pouvaient être appliqués sans plus aux résultats des recherches sur les affouillements tridimensionnels, à condition que ces recherches aient été effectuées sur un modèle normal. Pour vérifier cela, on fait actuellement des contrôles au moyen de l'affouillement du

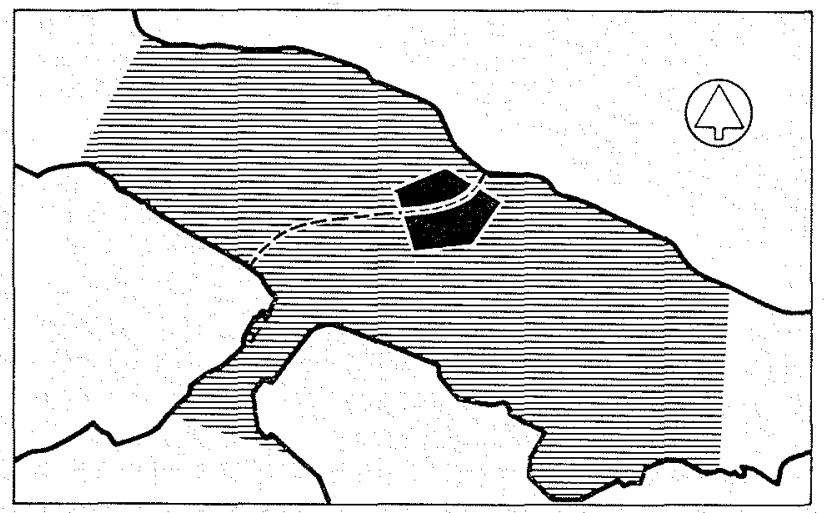

MODEELE DE DÉTAIL DISTORDU ECHELLE 1/50

\section{MODELE GÉNÉRAL} ECHELLE $\left\{\begin{array}{l}\text { HORIZONTALE } 1 / 150 \\ \text { VERTICALE 1/60 }\end{array}\right.$

Fig. 20 Grevelingen.
Veerse Gat, qui est reproduit encore une fois dans un nouveau modèle à l'échelle normale, mais cette fois de telle façon que l'écoulement exact soit imposé au modèle dans son ensemble. Dans le modèle original, cela n'était pas possible parce que les limites du modèle étaient trop éloignées l'une de l'autre. En se basant sur les expériences vécues que j’ai rapportées brièvement, on a décidé, pour les recherches à effectuer sur les prochaines passes à fermer, qu'un modèle à distorsion ne serait construit que pour l'étude de l'écoulement et surtout de l'emplacement des chapelets de tourbillons. L'étude de l'affouillement pourra se faire sur un modèle non distordu, dans lequel on imposera cependant l'écoulement du modèle à distorsion. Un avantage de cette méthode est qu'à présent nous n'avons plus qu'un seul modèle qui doit être étalonné sur le prototype.

Cette méthode de travail est appliquée en ce moment dans les recherches destinées à la passe de fermeture septentrionale dans le Grevelingen (fig. 20). Au début de ma causerie, j'ai montré quelques résultats des recherches sur les affouillements dans le modèle réduit non distordu. La configuration de l'écoulement imposée à ce modèle a été obtenue dans un modèle d'ensemble qui représente une surface beaucoup plus grande à l'échelle horizontale de 1:150 et à l'échelle verticale de 1:60. Des expériences sur les affouillements seront encore effectuées dans ce modèle à distorsion, particulièrement en ce qui concerne l'affouillement provoqué par le 
PROTOTYPE

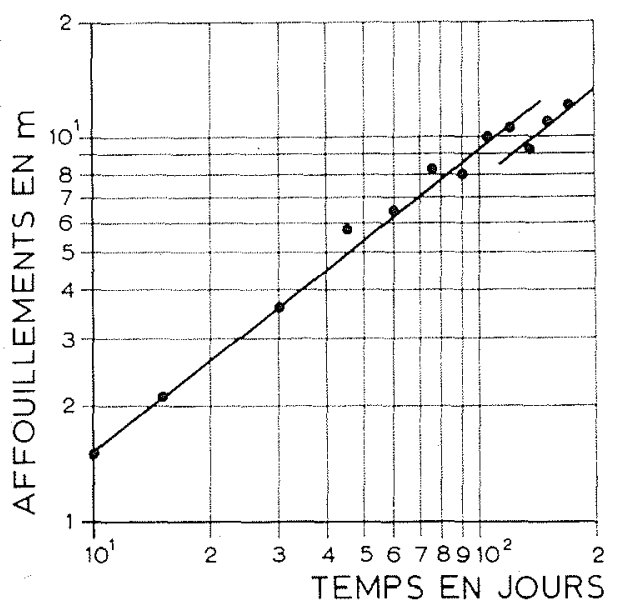

MODE்LE RÉDUIT

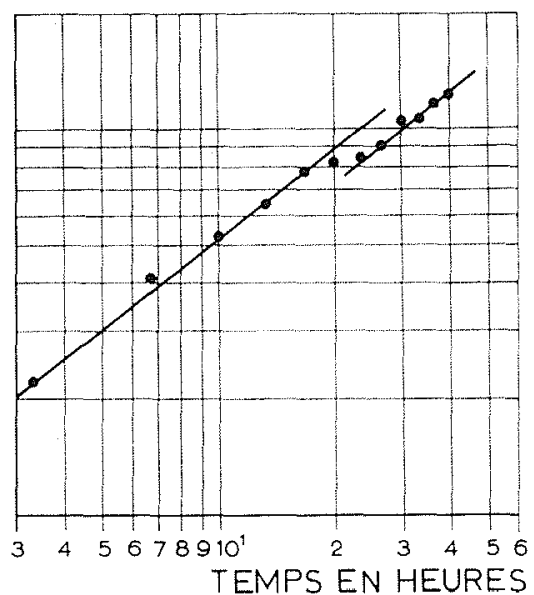

Frg. 21

Veerse Gat. Comparaison des affouillements en prototype et en modèle réduit.

chapelet de tourbillons dans la passe de fermeture septentrionale. Entre-temps, nous nous sommes fait une idée de l'échelle du temps dans ce modèle en reproduisant l'affouillement de la passe de fermeture méridionale.

Pour la passe de fermeture septentrionale, nous disposons donc de deux données. Sur la base de ces données, nous pouvons émettre une opinion sur les affouillements qui se produiront cet hiver. Au cours de cet hiver, les résultats pourront être confrontés avec les observations faites dans le prototype. Ainsi nous espérons pouvoir restreindre encore le problème posé par l'échelle du temps.

Pour terminer, je vais encore attirer l'attention sur un autre aspect intéressant des affouillements produits par les chapelets de tourbillons. Lorsque j'ai parlé de la reproduction de l'affouillement dans le Veerse Gat, j'ai montré l'évolution du point le plus profond en fonction du temps. Sur papier logarithmique, une ligne droite donne une bonne approximation de cette fonction. Il apparait maintenant que les fonctions, par rapport au temps de l'affouillement consécutif à un chapelet de tourbillons dans les diverses autres situations que nous avons étudiés et dont j’ai donné quelques exemples, suivent la même pente (fig. 21). Cela pourrait indiquer que lorsqu'un chapelet de tourbillons provoque un affouillement, il le fait suivant cette pente. Une recherche systématique sera encore nécessaire pour vérifier si cela s'aplique d'une manière générale. S'il en était ainsi, cela signifierait que, dépendant de l'intensité du chapelet de tourbillons, la droite représentative se déplace soit vers le haut, soit vers le bas.
Il est intéressant de comparer cette pente avec celle qui a été définie lorsqu'on s'est basé sur la recherche systématique de l'affouillement bidimensionnel (fig. 22). On voit donc que la pente due au chapelet de tourbillons est environ deux fois plus raide, ce qui veut tout simplement dire que si un chapelet de tourbillons provoque un affouillement, il creuse un trou profond beaucoup plus vite que ne le ferait un courant bidimensionnel. Une fois de plus, c'est une raison d'accorder une attention toute particulière, pour chaque passe de fermeture, aux effets tridimensionnels, auxquels on doit s'attendre aux limites de ces passes.

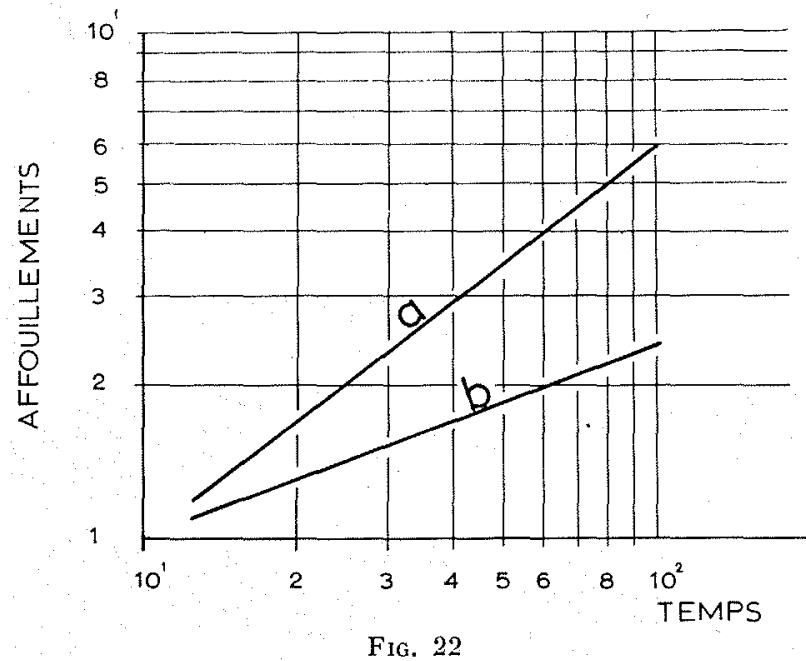

Comparaison des fonctions des affouillements par rapport au temps.

a) Comme suite des chapelets de tourbillons;

b) L'écoulement bi-dimensionnel. 


\section{DISCUSSION}

Président : M. De Rovville

M. le Président remercie M. Venis pour sa communication.

M. le Président a remarqué que, ni dans la communication de M. Bischoff Van Heemskirck, ni dans celle de M. Venas, il n'a été question de gabions de sable qui ont paru très ingénieux. $Y$ a-t-on recouru pour mieux asseoir les caissons, par exemple?

M. Bischoff Van HeEasherci indique que le Service du Delta utilise des sacs de nylon à parois très minces dans lesquels on crée une sous-pression qui soumet le sable à l'intérieur à une précontrainte de compression. Ces sacs ont un prix de revient très avantageux et on peut les utiliser pour n'importe quel seuil.

M. Charon pense qu'il serait intéressant que les ingénieurs néerlandais donnent leur avis sur l'emploi des tapis de nylon (lestés par des boudins de sable) et des revêtements du fond en mastic de bitume, pour lutter contre les affouillements au voisinage des seuils déversants ou dans les brèches avant fermeture complète des digues.

M. Bischoff Van Heemskenck indique que l'on emploie des tapis très minces, soit en nylon perméable, soit en polystyrène imperméable sous formes différentes. Ces tapis sont lestés, soit par des boudins remplis de sable, soit par des enrochements, dans le but d'empêcher qu'ils se mettent à rouler. Quant aux revêtements de fond en asphalte, bien souvent le calcul des couches mène à des épaisseurs onéreuses. Aussi, n'applique-t-on l'asphalte que lorsqu'une faible épaisseur suffit. Ces protections servent à éviter tout simplement que le sable soit érodé.

M. le Président s'étonne d'entendre parler de procédés trop conteux pour des travaux considérables et vitaux comme ceux-ci; mais n'ayant pas fait les calculs, il s'incline devant l'avis du Service du Delta.

M. Bischoff Van Heemsierci dit que lorsqu'on utilise une méthode chère, le surplus de dépense est énorme, car les travaux s'étendent sur 8 kilomètres.

M. Grraudet pose les questions suivantes :

$1^{0}$ Quelle est la vitesse d'érosion des massifs de sable stabilisés au bitume, avec faible dosage de bitume (3 à $4 \%$ )?

$2^{\circ}$ Pour les écoulements sous les digues dans les sables de fondation, a-t-on étudié la perméabilité in situ de ces sables ef, éventuellement, par quel procédé?

M. Bischoff VAN HEemskenck, répondant à la première question, précise qu'il est difficile de donner une réponse d'une façon générale du fait que l'on ne dispose que d'un nombre limité d'essais qui, à leur tour, ont été effectués par des méthodes diverses. De toute facon, nous donnons ici quelques résultais qui se rapportent à un fond plan à une profondeur de $0,50 \mathrm{~m}$.

$\begin{array}{cc}\text { Dosage } & \begin{array}{c}\text { Vitesse critique } \\ \text { (d'érosion) }\end{array} \\ 8,5 \% & - \\ 5,5 \% & 1,4 \mathrm{~m} / \mathrm{s} \\ 3,5 \% & 1,1 \mathrm{~m} / \mathrm{s} \\ & 1,0 \mathrm{~m} / \mathrm{s}\end{array}$

Il est à remarquer que ces figures vont en s'améliolant après une période plus ou moins prolongée de repos.

M. Bischork VAN HeemskencK répond à la deuxième question de M. Grrauder que la perméabilité ne joue pas tant que la capacité d'absorption du corps de la digue lui-meme n'intervient pas. Si l'on a affaire à une protection de fond ee cas ne se présente que rarement. D'autre part, pour la détermination des sous-pressions sollicitant les revètements d'une digue la connaissance de la perméabilité est de toute importance.

le graphique A ei-eontre représente la variation des sous-pressions en fonction de la perméabilité dans une certaine digue aux Pays-Bas. Dans le cas dont il s'agit la vraie perméabilité varie de $5.10-4$ à $10-5 \mathrm{~m} / \mathrm{s}$ environ.

M. le Président est étonné d'avoir vu, dans une des figures projetées au cours du rapport de M. Venis, des vitesses plus grandes quand il n'y avait pas de tourbillons ( $7 \mathrm{~m}$ ) que Ior'squ'il y en avait $(3,50 \mathrm{~m})$.

M. Venis explique que, à la suite des essais sur modèle relatifs à la stabilité des matériaux de construction des seuils, on a trouvé que, dans certains cas, avec un courant bi-dimensiomel dans l'axe de la passe, un commencement d'affouillement se manifestait à, disons, $7 \mathrm{~m} / \mathrm{s}$. Si l'on place sur un tel seuil un caisson plein qui engendre des chapelets de tourbillons, on s'aperçoit que

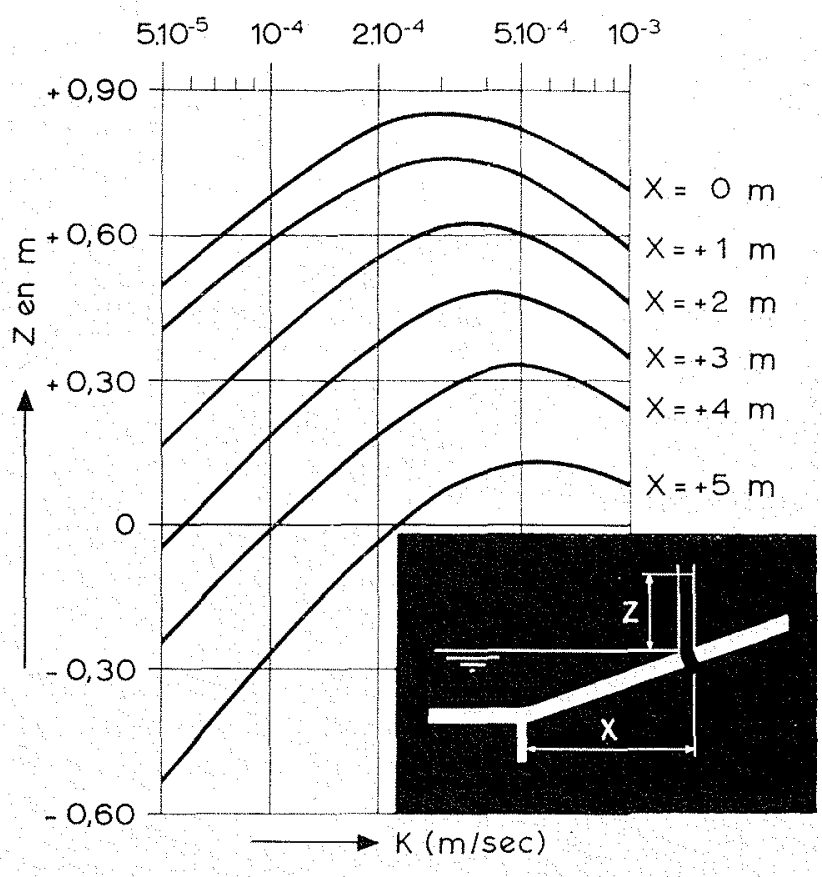

Croouis A

Pression hydrostatique au moment de la surpression maximale par rapport à la surface supérieure du revêtement en fonction du coefficient de perméabilité Is. 

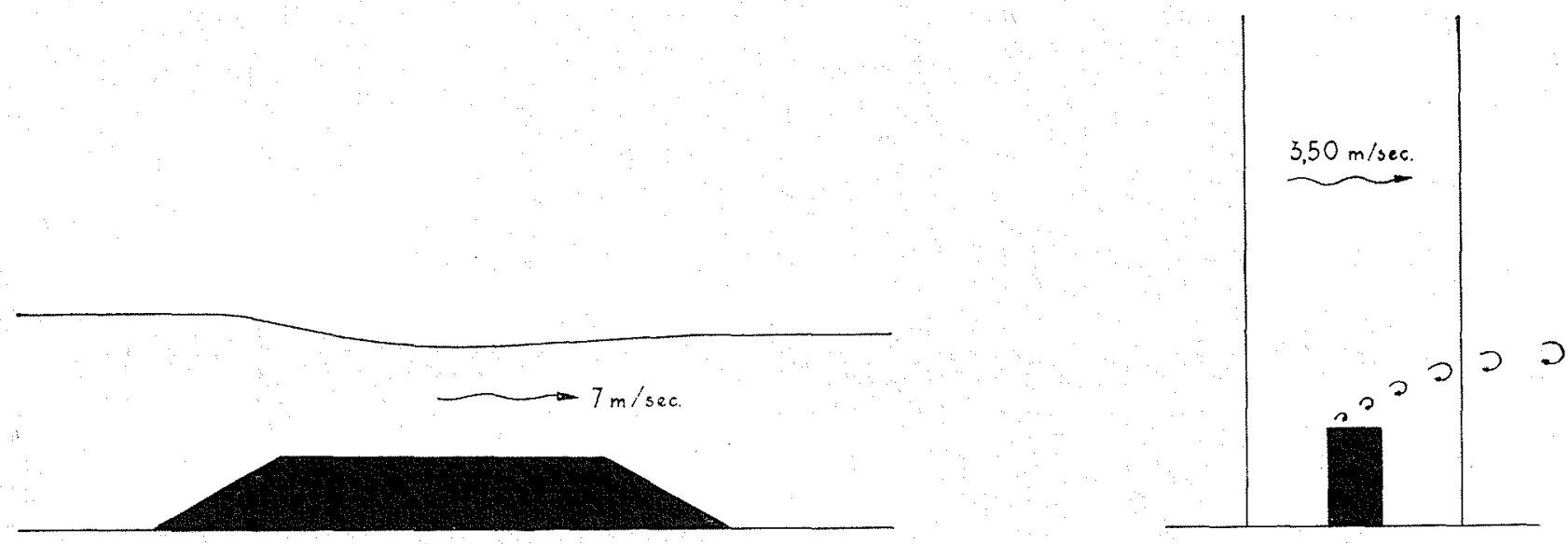

Croovis $\mathrm{B}$

les matériaux qui sont assaillis par ces tourbillons sont emportés du moment que la vitesse dans l'axe de la passe est de $3,50 \mathrm{~m}$ seulement, donc réduite de moitié. Cette aggravation des valeurs critiques de la vitesse d'érosion dépend de l'intensité du chapelet de tourbillons.

M. DE FERIET aimerait savoir si, dans la nature, il a été possible de mesurer les périodes des tourbillons dont on a parlé. On a très peu de renseignements sur ces tourbillons pour les obstacles de très grandes dimensions.

M. Schommaken indique que, dans la nature, il est difficile d'obtenir des résultats et que les expériences effectuées sur le Veerse Gat par le Service du Delta, en collaboration avec le Laboratoire de Delft, n'ont pas eu de grands sucè̀s.
On a utilisé des moulinets de réaction très rapides, mis au point par le «Rijkswaterstaat», qui mesurent les composantes de vitesses. De mème, on a employé l'appareil mis au point par le «Waterlooplundig Laboratorium $\gg$; avec lequel on mesure en même temps deux composantes mutuellement perpendiculaires de la vitesse. Mais les difficultés dans la pratique (câbles de connexion, profondeur d'eau de $30 \mathrm{~m}$, courants très forts) ont été tellement considérables que les résultats ne sont pas fidèles. On a essayé de mesurer ces variations dans le modèle dont va parler M. Privs; mais la comparaison avec la nature n'est pas encore définitive.

M. le Président remercie encore M. VENIs pour son exposé.

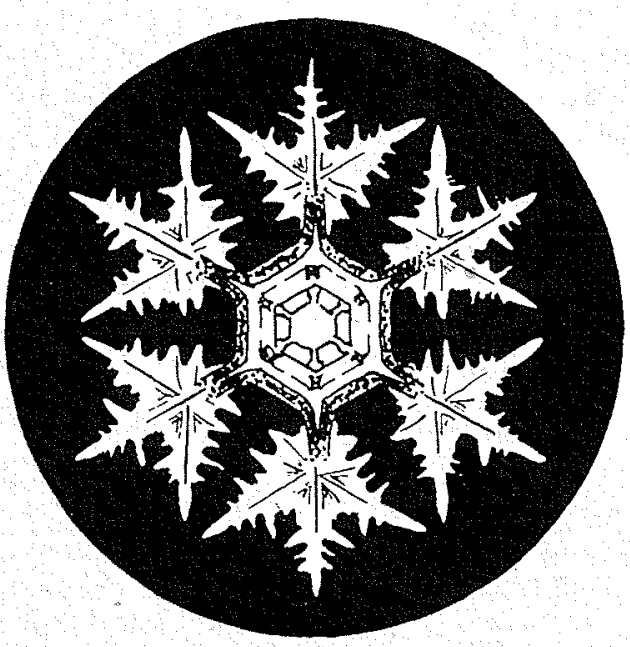

